\section{New recommendations from the United States Government on breast cancer screening ${ }^{1}$}

Key words: Breast cancer, women's health, screening, guidelines.

\footnotetext{
1 Based on: United States of America, Agency for Healthcare Research and Quality. Screening for breast cancer. Recommendations and rationale. Rockville, Maryland, United States of America: AHRQ; 2002. Available from: http://www.ahrq.gov/clinic/3rduspstf/breastcancer/ brcanrr.htm [Internet site]. Accessed 22 February 2002.
}

In recent years there has been much controversy and debate concerning the effectiveness of various approaches to screening for breast cancer. Even within the Government of the United States of America, various agencies and expert commissions have disagreed with each other. Now, in what Tommy G. Thompson, the Director of the Department of Health and Human Services (HHS) of the United States has termed the "final word" on breast cancer screening $(1,2)$, an expert panel has affirmed that mammography does save lives and that it should begin for women in their forties. The panel also said that there is insufficient evidence to either recommend for or against two other screening methods, clinical breast examination and routine breast self-examination.

In 1998 the HHS's Agency for Healthcare Research and Quality asked the expert panel, called the U.S. Preventive Services Task Force (USPSTF), to look at the controversial issue and to update recommendations that the Task Force had made in 1996 (3). For their new recommendations the USPSTF reviewed the evidence concerning the effectiveness of mammography, clinical breast examination (CBE), and breast self-examination (BSE) in reducing breast cancer mortality. A meta-analysis using a Bayesian random effects model was used to obtain a summary of relative risk estimates of the effectiveness of screening with mammography, either alone or in combination with clinical breast examination, in reducing breast cancer mortality.

The USPSTF looked at many of the same studies that have been assessed by two Danish researchers who have concluded that mass screening for breast cancer is not beneficial $(4,5)$. However, the assessment by the two Danes has generated strong controversy, with many other scientists challenging their conclusions $(6,7)$. While acknowledging that there were flaws in many of the screening studies that they and the Danish researchers had looked at, the USPSTF panel did not consider the methodological limitations to be "fatal" (1). The USPSTF concluded that there is a "fair" level of evidence that regular mammograms can significantly reduce the chances of dying from breast cancer. 


\section{SCIENTIFIC EVIDENCE}

\section{Epidemiology and clinical consequences in the United States}

In the United States, breast cancer is the most common nonskin malignancy among women and second only to lung cancer as a cause of cancerrelated death. In 2001 an estimated 192200 new cases of breast cancer were diagnosed in women in the country, and 40200 women died of the disease. The risk of developing breast cancer increases with age, beginning in the fourth decade of life. The probability of developing invasive breast cancer over the next 10 years is $0.4 \%$ for women aged $30-39,1.5 \%$ for women aged $40-49,2.8 \%$ for women aged $50-59$, and $3.6 \%$ for women aged 60-69. Individual factors other than age that increase the risk for developing breast cancer include family history or a personal history of breast cancer, biopsy-confirmed atypical hyperplasia, and having a first child after age 30 .

\section{Accuracy and reliability of screening tests}

The USPSTF examined the test characteristics of mammography, CBE, and BSE. Precise estimates of sensitivity and specificity of screening are made more difficult by the varied criterion standards in available studies. Estimating the predictive value of positive and negative tests is also difficult because studies have been conducted on populations with a widely varying prevalence of breast cancer.

Estimates of the sensitivity of mammography vary with the methods used to calculate it. In one good-quality systematic review, the first round of mammography detected $77 \%$ to $95 \%$ of cancers diagnosed over the following year, but only $56 \%$ to $86 \%$ of cancers diagnosed over the next 2 years. Sensitivity is lower among women who are younger than 50 (51\% to $83 \%)$, have denser breasts, or are taking hormone replacement therapy.

In screening trials, the false-positive rate of the initial round of mammography was $3 \%$ to $6 \%$ (i.e., specificity of $94 \%$ to $97 \%$ ). Specificity is increased with a shorter screening interval and the availability of prior mammograms. In one large study in a health maintenance organization, the rate of false-positive mammograms (those requiring some additional follow-up) was higher in women aged $40-59(7 \%$ to $8 \%)$ than in women aged $60-79$ $(4 \%$ to $5 \%)$.

The probability that an abnormal mammogram is due to cancer increases with age. One large study in California estimated positive predictive values for abnormal mammograms at $2 \%$ to $4 \%$ among women aged $40-49,5 \%$ to $9 \%$ among women aged $50-59$, and $7 \%$ to $19 \%$ among women aged 60 and older. Positive predictive values were also higher among women with a family history of breast cancer in two studies that the USPSTF considered.

With clinical breast examination, in one recent good-quality review of data from clinical trials, the sensitivity of CBE ranged from $40 \%$ to $69 \%$, specificity from $86 \%$ to $99 \%$, and positive predictive value from $4 \%$ to $50 \%$, using mammography and interval cancer as the criterion standard. In one large community study, only $4 \%$ of women with an abnormal CBE were subsequently diagnosed with cancer.

The accuracy of breast self-examination is largely unknown. Available evidence shows sensitivity ranging from $26 \%$ to $41 \%$ as compared with CBE and mammography. Specificity of BSE is largely unknown.

\section{Effectiveness of early detection}

The USPSTF reviewed eight randomized controlled trials (RCTs) of mammography (four of mammography alone and four of mammography plus CBE) that have reported results with 11-20 years of follow-up. The USPSTF found important methodological limitations in each trial, but rated only one trial as "poor" based on established criteria used by the USPSTF to evaluate the quality of evidence for screening tests. The USPSTF did not consider the presence of the flaws as sufficient reason in itself for rejecting the trials' results. Instead, the USPSTF examined whether observed mortality reductions in the trials were likely to be explained by the biases potentially introduced by such flaws.

The trials reported mortality reductions ranging from no significant effect to a $32 \%$ reduction in breast cancer mortality. The meta-analysis performed for the USPSTF on the most current published data found that the pooled effect size of the combined trials was sizable and statistically significant: the summary relative risk (RR) of breast cancer death among women randomized to screening in seven trials that included women older than 50 was 0.77 (95\% confidence interval (CI) of 0.67-0.89). Eliminating one trial considered to be of poor quality and one trial that lacked a usual-care control group did not noticeably change the results $(R R=0.75 ; 95 \% C I, 0.63-0.89)$. Similar results were observed in the four trials of mammography alone $(\mathrm{RR}=0.74 ;$ 95\% CI, 0.59-0.93).

Earlier subgroup analyses from mammography trials raised questions about whether screening is effective in women younger than 50 . Seven trials enrolled women aged 40-49. While six of these were 


\section{Amid new technologies for breast cancer detection, film mammography remains the gold standard}

Although several new technologies under development show promise for improved capability to detect breast cancer, none has yet proved superior to traditional, X-ray film mammography in screening for breast cancer, according to a comprehensive report issued in March 2001 by the Institute of Medicine (IOM) and National Research Council of the National Academies of the United States of America.

More evaluation and development of new imaging tools and of promising molecular biological techniques is required and warranted, according to the study, which is entitled Mammography and Beyond: Developing Technologies for the Early Detection of Breast Cancer. No single imaging technology is capable of accurately detecting all breast abnormalities. Ultimately, the report says, the best detection may come from using several different tools. For example, ultrasound and magnetic resonance imaging have shown potential as adjuncts to mammography in diagnosis and screening, especially in getting a clearer picture of dense breast tissue in certain women.

In addition to evaluating scientific evidence on the new technologies, the report examines the process by which newer screening technologies move from testing to routine clinical usage. The report raises a concern that technologies approved for diagnosis could be prematurely adopted for screening, noting that diagnostic tools help determine the nature of a breast abnormality first detected through screening and may not be appropriate for both purposes. In evaluating a new technology's appropriateness for screening, the Food and Drug Administration (FDA) and the Health Care Financing Administration of the United States should base their approval and coverage decisions on the results of clinical trials that prove screening effectiveness, the report says.

While imaging technologies indicate structural differences or changes in the breast, such as microcal- cifications or new growths, some of the latest molecular biological technologies can provide information about the cellular characteristics of these abnormalities and thus potentially lead to more accurate screening and diagnosis. These newer approaches include growing breast cancer cells in the lab and identifying the genetic changes in particular kinds of tumors.

The report looks at and evaluates film mammography and 17 other imaging tools that are currently available. These includes ones with FDA approval, such as full-field digital mammography, ultrasound, computer-aided detection systems, and magnetic resonance imaging, as well as those not yet approved, such as optical imaging. Many appear to offer varying degrees of potential for screening or diagnosis-in some cases, both-but more research is needed. No studies have shown a new technology to be a replacement for film mammography, for either screening or diagnosis. For instance, while digital mammography has been lauded as a major technical advance-facilitating storage, retrieval, transmission, and image adjustment for mammograms-it has not shown greater accuracy than its nondigital counterpart.

Earlier identification of breast-tissue abnormalities will remain problematic, the report says, until a deeper understanding of the biology and genetics of such abnormalities makes it possible to distinguish those that are nonthreatening from those that may become invasive and progress to full-blown, metastatic breast cancer.

The complete report on technologies for breast cancer detection can be purchased from the National Academy Press (NAP), 2101 Constitution Avenue, N.W., Lockbox 285, Washington, D.C. 20055, United States of America, telephone (202) 334-3313 or (800) 624-6242. The report can also be purchased from the NAP Web site (http://www.nap.edu), which also offers free page-by-page viewing of the full document. rated by the USPSTF to be of at least "fair" quality, only one of them was designed to specifically address the benefits of screening in this age group. That one study reported no reduction in breast cancer mortality with annual mammography and CBE. Of the remaining five fair-quality trials that included women younger than 50, two trials have now reported significant mortality reductions with screening in this age group, two have reported nonsignificant mortality reductions, and one found no benefit.

In a meta-analysis performed for the USPSTF that pooled results for women aged 40-49 in these six trials, the relative risk of breast cancer mortality 
was 0.83 (95\% CI, 0.64-1.04) among screened women; inclusion of the seventh, poor-quality study did not change the results.

Because these data represent a subgroup analysis of trials not designed to test the benefits of beginning screening at a specific age, questions remain about the additional benefits of beginning screening before age 50 . On average, the time until mortality benefits begin to be observed in these trials is longer in women younger than 50 than in older women (8 years vs. 3 to 4 years), and some of the observed benefits could be due to screening after age 50. Analyses of individual studies suggest that at least some of the mortality reduction is due to early detection of tumors before age 50 , but definitive estimates of the proportion of benefits due to early screening cannot be made.

With respect to clinical breast examination, no study has compared CBE to no screening. The reductions in breast cancer mortality in studies using mammography alone are comparable to those using mammography plus CBE.

The role of BSE in reducing breast cancer mortality has been evaluated in one Chinese and one Russian RCT and in one nonrandomized controlled trial of BSE education in the United Kingdom. None of the three trials has demonstrated a reduction in breast cancer mortality or significant improvements in the number or stage of cancers detected, with follow-up ranging from 5 to 14 years; follow-up is continuing in one trial that observed a slight nonsignificant reduction in mortality in the BSE group at 9 years. In a good-quality nested casecontrol analysis from a Canadian screening study, the overall practice of BSE was not associated with a reduction in mortality.

Although none of these studies provides support for BSE, the USPSTF concluded that these studies did not exclude a possible benefit, due to their limited duration of follow-up and questions about whether results from other countries are generalizable to women in North America.

\section{CLINICAL CONSIDERATIONS}

The precise age at which the benefits from screening mammography justify the potential harms is a subjective judgment and should take into account patient preferences. Clinicians should inform women about the potential benefits (reduced chance of dying from breast cancer), potential harms (e.g., false-positive results, unnecessary biopsies), and limitations of the test that apply to women their age. Clinicians should tell women that the balance of benefits and potential harms of mammography improves with increasing age for women between the ages of 40 and 70 .

Women who are at increased risk for breast cancer (e.g., those with a family history of breast cancer in a mother or sister, a previous breast biopsy revealing atypical hyperplasia, or first childbirth after age 30) are more likely to benefit from regular mammography than women at lower risk. The recommendation for women to begin routine screening in their forties is strengthened by a family history of breast cancer having been diagnosed before menopause.

In the trials that demonstrated the effectiveness of mammography in lowering breast cancer mortality, screening was performed every 12-33 months. For women aged 50 and older, there is little evidence to suggest that annual mammography is more effective than mammography done every other year.

For women aged 40-49, available trials also have not reported a clear advantage of annual mammography over biennial mammography. Nevertheless, some experts recommend annual mammography, based on the lower sensitivity of the test and on evidence that tumors grow more rapidly in this age group.

The precise age at which to discontinue screening mammography is uncertain. Only two randomized controlled trials enrolled women older than 69 , and no trials enrolled women older than 74 . Older women face a higher probability of developing and dying from breast cancer but also have a greater chance of dying from other causes.

Clinicians who advise women to perform BSE or who perform routine CBE to screen for breast cancer should understand that there is currently insufficient evidence to determine whether these practices affect breast cancer mortality, and that they are likely to increase the incidence of clinical assessments and biopsies.

\section{Potential harms of screening}

Similar to other cancer screening tests, the large majority ( $80 \%$ to $90 \%$ ) of abnormal screening mammograms or CBEs are false-positives. These may require follow-up testing or invasive procedures such as breast biopsy to resolve the diagnosis, and can result in anxiety, inconvenience, discomfort, and additional medical expenses.

In one large community study, $6.5 \%$ of screening mammograms required some additional follow-up and, over a 10-year period, $23 \%$ of all women had experienced at least one abnormal mammogram. The cumulative risk of a false-positive re- 
sult after 10 mammograms was estimated to be $49 \%$. The proportion of false-positive results that lead to biopsy varies substantially in different settings. In screening trials, $1 \%$ to $6 \%$ of all women screened underwent biopsy, and the proportion of biopsies that revealed cancer ranged from $12 \%$ to $78 \%$. In two RCTs, BSE education resulted in a nearly two-fold increase in false-positive results, physician visits, and biopsies for benign disease.

The consequences of false-positive mammograms are uncertain. Most, but not all, studies report increased anxiety from an abnormal mammogram. At the same time, some studies report that women in the United States may be willing to accept a relatively high number of false-positive results in the population in return for the benefits of mammography. Studies do not indicate that falsepositive results diminish adherence to subsequent screening.

False-negatives also occur with mammograms and $\mathrm{CBE}$. Although false-negative results might provide false reassurance, the USPSTF found no data indicating these led to further delays in diagnosis.

\section{RECOMMENDATIONS}

Based on all the evidence that the panel examined, the USPSTF reached three overall conclusions:

- screening mammography, with or without clinical breast examination, is recommended every 1-2 years for women aged 40 and older
- the evidence is insufficient to recommend for or against routine clinical breast examination alone to screen for breast cancer

- the evidence is insufficient to recommend for or against teaching or performing routine breast self-examination

\section{SINOPSIS}

\section{Nuevas recomendaciones del Gobierno de los Estados Unidos sobre el tamizaje del cáncer de mama}

En los últimos años ha habido grandes controversias y debates sobre la eficacia de diferentes métodos de detección del cáncer de mama. En un esfuerzo por proporcionar orientaciones más claras tanto a las mujeres como a los profesionales de la salud, un grupo de expertos reunido por el Gobierno de los Estados Unidos de América, el llamado U.S. Preventive Services Task Force (USPSTF), publicó en febrero de 2002 un informe en el que se afirma que la mamografía permite salvar vidas y que su realización debería comenzar en la quinta década de la vida de la mujer. Este grupo de expertos dice también que no hay datos suficientes para hacer recomendaciones a favor o en contra de otros dos métodos de detección: el examen clínico de la mama y la autoexploración mamaria rutinaria. El presente documento resume las recomendaciones del informe del USPSTF, así como las principales consideraciones clinicas y pruebas científicas que presentó en áreas como la exactitud y fiabilidad de las pruebas de detección, la eficacia de la detección temprana del cáncer de mama, cuándo detener el tamizaje, el intervalo de tiempo más apropiado entre las pruebas y los potenciales perjuicios del tamizaje.

\section{REFERENCES}

1. Garvey M. U.S. says mammograms help, and that's final. Los Angeles Times 22 February 2002. Available from: http:// www.latimes.com [Internet site]. Accessed 23 February 2002

2. Trafford A. 'Final word' on mammograms? Not yet. Washington Post 5 March 2002; HE1.

3. United States, Preventive Services Task Force. Guide to clinical preventive ser- vices, second edition. Washington, D.C.: Office of Disease Prevention and Health Promotion; 1996

4. Gøtzsche PC, Olsen O. Is screening for breast cancer with mammography justifiable? Lancet 2000;355(9198):129-134.

5. Olsen O, Gøtzsche PC. Cochrane review on screening for breast cancer with mammography. Lancet 2001;358(9290): 13401342.
6. Duffy SW, Tabar L. Screening mammography re-evaluated [letter]. Lancet 2000; 355(9205):747-748.

7. The CBCG Editors. Screening mammography: setting the record straight [letter]. Lancet 2002;359(9304):439-440. 